\title{
Esclerose múltipla e tratamentos
}

\author{
Dr. Fabiano de Abreu Agrela Rodrigues ${ }^{1}$ \\ deabreu.fabiano@gmail.com
}

Henry $\mathrm{Oh}^{2}$

\section{RESUMO}

A esclerose múltipla é uma patologia autoimune que atinge o cérebro, bem como seus nervos ópticos e a medula espinhal. Quando a bainha de mielina está em seu estado normal e saudável, os sinais nervosos são enviados e recebidos rapidamente. Porem quando o indivíduo possuí a Esclerose Múltipla, o sistema imunológico do seu corpo trata a mielina como uma ameaça, atrapalhando o envio dos comandos do cérebro até o restante do corpo, denominado como desmielinização. O objetivo do presente estudo foi compreender a formação da esclerose múltipla, seus danos a bainha de mielina e os possíveis tratamentos da doença. O presente estudo é uma revisão de literatura, realizada por meio das bases de dados: SciELO, PubMed e Science Direct. Os tratamentos para a esclerose múltipla são a base de corticoides, atividade física, alimentos anti-inflamatórios e ricos em gorduras insaturadas. O elemento lítio vem sendo estudado e demonstrando efeitos benéficos, sendo um tratamento eficaz para esta patologia.

Palavras-chave: esclerose múltipla; doenças autoimunes; neurociência; bainha de mielina

\footnotetext{
${ }^{1} \mathrm{PhD}$, neurocientista, mestre em psicanálise, biólogo, historiador, antropólogo, com formações também em neuropsicologia, neurolinguística, neuroplasticidade, inteligência artificial, neurociência aplicada à aprendizagem, filosofia, jornalismo, programação em python e formação profissional em nutrição clínica Diretor do Centro de Pesquisas e Análises Heráclito; Chefe do Departamento de Ciências e Tecnologia da Logos University International e da City University; Membro da SFN - Society for Neuroscience, Membro Redilat.
}

2 Cientista e biólogo certificado no Reino Unido, terapeuta respiratório e tecnólogo médico certificado nos EUA, Membro da Associação de Cientistas Clínicos e Chefe do departamento de saúde da Faculdade de Tecnologia da Universidade Estadual de Idaho nos Estados Unidos. 


\title{
Multiple Sclerosis and Treatments
}

\begin{abstract}
Multiple sclerosis is an autoimmune condition that affects the brain, as well as its optic nerves and spinal cord. When the myelin sheath is in its normal, healthy state, nerve signals are sent and received quickly. However, when the individual has Multiple Sclerosis, the body's immune system treats myelin as a threat, and hinders the sending of commands from the brain to the rest of the body, called demyelinating. The aim of the present study was to understand the formation of multiple sclerosis, its damage to the myelin sheath and possible treatments for the disease. This study is a literature review, carried out through the following databases: SciELO, PubMed and Science Direct. Treatments for multiplying sclerosis are based on steroids, physical activity, antiinflammatory foods and foods rich in unsaturated fats. The element lithium has been studied and showing beneficial effects, being an effective treatment for this pathology.
\end{abstract}

Keywords: multiple sclerosis; autoimmune diseases; neuroscience;myelin sheath.

Artículo recibido: 02 enero 2022 Aceptado para publicación: 28 enero 2022 Correspondencia: deabreu.fabiano@gmail.com Conflictos de Interés: Ninguna que declarar 


\section{Henry Oh}

\section{INTRODUÇÃO}

\subsection{Esclerose múltipla}

É uma patologia autoimune que atinge o cérebro, bem como seus nervos ópticos e a medula espinhal. No Brasil, estima-se que surgem 40.000 casos, com uma incidência média de 15 por 100.000 habitantes, sendo em sua maioria jovens entre 20 e 40 anos de idade, principalmente mulheres (Katz, 2015).

Os principais fatores de risco, podem estar relacionados a genética. Porém, fatores ambientais tais como: Infecções virais (herpes vírus ou retrovírus); Exposição ao sol insuficiente, o que leva a ter níveis baixos de vitamina $\mathrm{D}$ por tempo prolongado; Exposição a solventes orgânicos; Tabagismo e Obesidade, também podem influenciar (Katz, 2015).

Os sintomas variam de acordo com o estágio da doença. Os principais são: fadiga; dificuldade em andar e em se equilibrar, coordenação motora, problemas de visão como visão dupla, visão borrada e embasada, incontinência ou retenção urinária, dormência ou formigamento em diferentes partes do corpo; rigidez muscular e espasmos; problemas de memória, atenção e para assimilar informações (Díaz, 2019).

Tais sintomas podem aparecer e desaparecer periodicamente ou piorar de maneira progressiva, assim como nas demais doenças de caráter neurológico (Díaz, 2019).

Existem cinco diferentes tipos de esclerose múltipla, sendo elas: Esclerose múltipla remitente-recorrente (EMRR), equivale a 85\% dos casos. Possuí características fortes de ocorrer períodos de melhora denominadas de remissão e de piora que podem durar dias, semanas ou até anos. Ocorrendo geralmente nos primeiros anos da doença e sem sequelas. Metade dos indivíduos em média de 10 anos, desenvolvem a Esclerose Múltipla Secundária Progressiva (EMSP) (Díaz, 2019).

Indivíduos com a EMSP, não se recuperam totalmente de suas crises denominadas como recaídas, promovendo sequelas ao longo dos anos. Pode ocorrer perda visual definitiva, grande dificuldade para andar. A Esclerose múltipla primária progressiva (EMPP) é descrita por causar uma piora gradativa das crises da doença (Díaz, 2019).

A Esclerose Múltipla Progressiva com Surtos (EMPS) atua de maneira rápida, agressiva e compromete estruturas do cérebro (Dobson, 2019).

Portanto, o diagnóstico da patologia é realizado em duas etapas: Avaliação clínica, após o indivíduo apresentar os sintomas o médico irá avaliar os estímulos do sistema nervoso 
por meio de um teste físico. Como por exemplo que o paciente caminhe, testa alguns reflexos do corpo e analisa a estrutura dos olhos (retina e disco óptico) (Dobson, 2019). Por meio da ressonância magnética, o exame de imagem mostra zonas de desmielinização no cérebro e na medula espinhal, provocado pelo ataque do sistema imunológico (Dobson, 2019).

\section{2 - Bainha de mielina e a doença}

A Bainha de Mielina atua como capa protetora de tecido adiposo que protege suas células nervosas. Tais células, atuam no transporte de mensagens entre o seu cérebro e o resto do seu corpo (Liu, 2019).

Quando a bainha de mielina está em seu estado normal e saudável, os sinais nervosos são enviados e recebidos rapidamente. Porem quando o indivíduo possuí a Esclerose Múltipla, o sistema imunológico do seu corpo trata a mielina como uma ameaça atrapalhando o envio dos comandos do cérebro até o restante do corpo, denominado como desmielinização (Lemus, 2018; Stadelmann, 2019).

Após isso, os nervos presentes no interior da bainha de mielina podem ser danificados, deixando assim cicatrizes, chamadas de esclerose (Lemus, 2018; Stadelmann, 2019).

Figura 1 - Formação da esclerose múltipla e danos a bainha de mielina

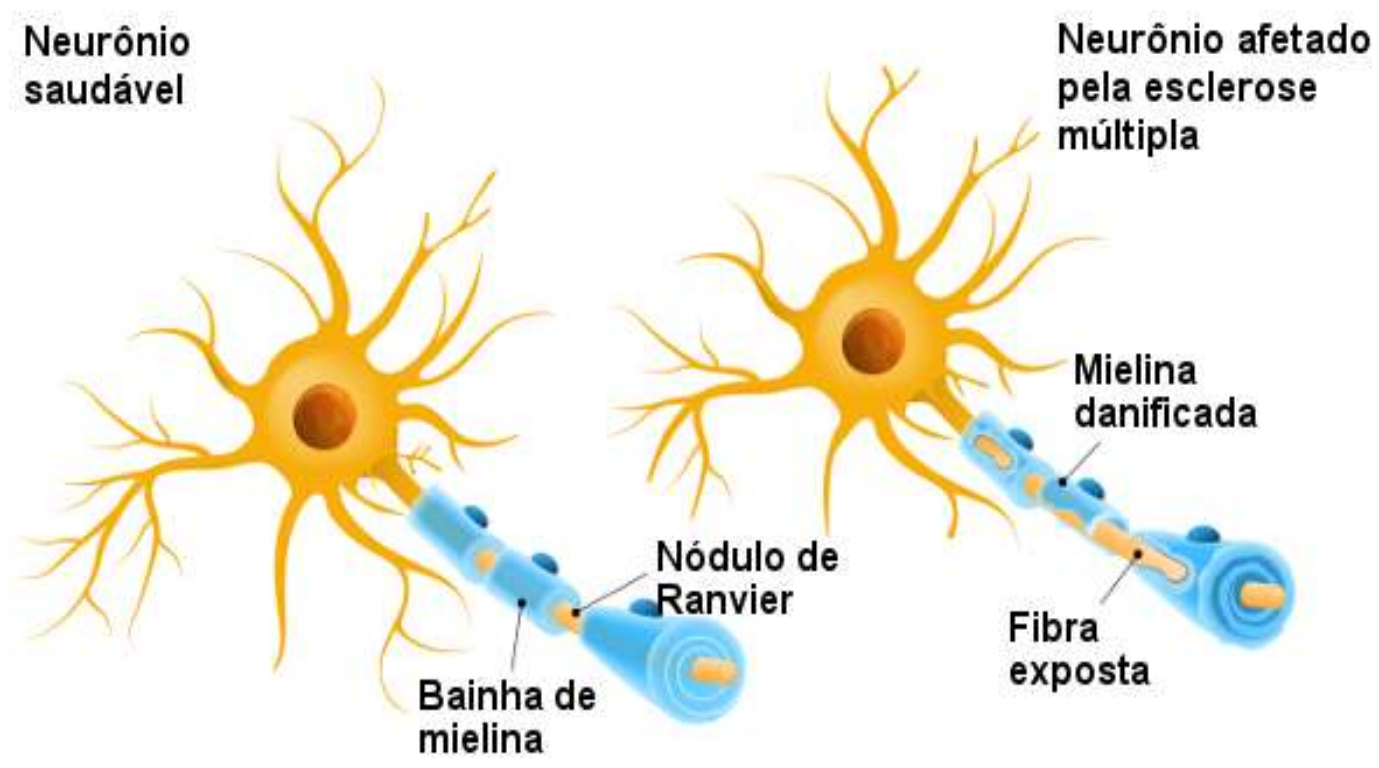

Fonte: Adaptado de Santos, et. al. 2019 
Henry Oh

2 OBJETIVO

Compreender a formação da esclerose múltipla, seus danos a bainha de mielina e os possíveis tratamentos da doença.

\section{METODOLOGIA}

O presente estudo é uma revisão de literatura, realizada por meio das bases de dados: SciELO, PubMed e Science Direct. Sendo utilizado os seguintes termos para realização da busca de dados em português: esclerose múltipla, doenças autoimunes, neurociência, bainha de mielina e em inglês: multiple sclerosis, autoimmune diseases, neuroscience, myelin sheath.

\section{RESULTADOS}

\subsection{Tratamentos}

Apesar de não haver cura para a esclerose múltipla, existe tratamento. Sendo o ideal começar o quanto antes para que o paciente tenha melhor qualidade de vida. (Doshi, 2016). Alguns dos tratamentos são:

Uso de corticoides, que auxiliam na inibição da ação do sistema imunológico. Na maioria das vezes, são administrados em curtos períodos para amenizar sintomas, como perda de visão, de força ou de coordenação. Podendo ser realizados de maneira oral ou injetável (Doshi, 2016).

Atividade física, pode auxiliar no tratamento como: pedalar, caminhar, nadar e se alongar, por exemplo, auxiliando na saúde cardiovascular, muscular e psicológica. Fisioterapia; auxiliando na capacidade de caminhar e o nível de mobilidade (Doshi, 2016).

Os ácidos graxos EPA (ácido eicosapentaenoico) e DHA (ácido docosa-hexaenoico) são importantes para formar adequada das membranas e reduzir o processo inflamatório da doença (Díaz, 2019).

Sendo assim, é importante o consumo de alimentos anti-inflamatórios, como açafrão da terra, frutas vermelhas (morango orgânico, framboesa, amora, cereja), gengibre e brássicas (brócolis, repolho, couve, couve-de-bruxelas, repolho). Alimentos ricos em gorduras insaturadas, como as sementes oleaginosas (amêndoas, nozes, castanhas), azeite de oliva extravirgem, linhaça, semente de chia e abacate (Morshedi, 2019).

Um relato de caso, de uma gravida com transtorno bipolar e esclerose múltipla, que fazia o uso de lítio demostrou a capacidade do lítio de inibir a glicogênio sintase quinase $3 \beta$, que é uma enzima atuante da fosforilação da Tau, proteína associada aos microtúbulos, 
estimulando uma possível melhora na doença de Alzheimer (Lorettu, 2020).

Em contrapartida, uma série de casos com 23 indivíduos que possuam a esclerose múltipla, demonstrando que o lítio não provocou efeitos colaterais e também não mostrou diferença significativa. Porém o estudo é limitado devido ao fato de possuir uma amostra populacional pequena e falta de grupo controle (Rinker, 2020).

Porém, no estudo de Karimi et. al. 2017, com 44 indivíduos com esclerose múltipla e 43 indivíduos saudáveis e sob o uso de lítio. Demonstrando aumento da produção de óxido nítrico em esclerose múltipla recorrente-remitente e níveis maiores de concentrações séricas de lítio no grupo controle do que nos demais (Karimi, 2017).

Um estudo de Oxford constatou que pessoas com doenças autoimune apresentam $20 \%$ de chances de sofrerem de doenças neurodegenerativas. Na esclerose múltipla este risco sobe para $97 \%$ de pacientes apresentarem doenças neurodegenerativas.

\section{CONSIDERAÇÕES FINAIS}

Compreendendo assim, o processo de formação da esclerose multiplica na bainha de mielina, bem como seus sintomas e diagnostico. Comumente diagnosticada em pacientes entre 20 e 40 anos, de maioria branca e principalmente mulheres, a Esclerose Múltipla (EM) é uma doença neurológica, crônica, progressiva e autoimune em que as células de defesa do nosso organismo atacam nossos próprios neurônios como se fossem patógenos causando lesões no cérebro e na medula espinhal. Estudos sugerem que a causa pode ter predominância genética, mas também o ambiente em que a pessoa vive ou vírus podem desempenhar um papel em seu desenvolvimento e a evolução varia de acordo com o indivíduo.

Os tratamentos para a esclerose multiplicam são a base de corticoides, atividade física, alimentos anti-inflamatórios e ricos em gorduras insaturadas. O elemento lítio vem sendo estudado e demonstrando efeitos benéficos, sendo um tratamento eficaz para tal patologia. Sugere-se estudos de ensaios clínicos randomizados, contendo grupo controle e amostra populacional grande, afim de compreender melhor os efeitos, mecanismos de ação do lítio na esclerose múltipla.

Pacientes com Esclerose Múltipla (EM) tem maiores probabilidades, chegando a média de 97\%, de manifestar déficits cognitivos e maior risco de apresentar doenças neurodegenerativas, como por exemplo a doença de Alzheimer. Sugerindo então atividades para a neuroplasticidade como leitura, jogos de lógica, treinamento cognitivo, 
Henry Oh

escrita, não fazer uso de substâncias químicas como drogas incluindo a maconha (Cannabis sativa), já que esta causa comprometimento no desempenho dos neurônios que resulta em atrofia e inativação que comprometem a inteligência, não fazer uso de álcool, cigarro, obrigando-se em manter uma vida saudável.

Há uma distribuição geográfica muito significativa na esclerose múltipla onde a doença é mais comum nos extremos dos hemisférios norte e sul do planeta e menos comum na linha equatorial, propondo assim que a vitamina $\mathrm{D}$ pode ser a razão destes números. $\mathrm{O}$ sol induz a IL-10 que inibe um pouco o sistema imunológico. Estudos clínicos comprovaram que a vitamina $\mathrm{D}$ potencializa o benefício do medicamento imunomodulador interferon- $\beta$, eficaz no tratamento de EM, funciona diminuindo a inflamação e prevenindo danos nos nervos, existindo uma sinergia entre a vitamina D e o interferon- $\beta$ retardando o progresso da doença.

Em caso de pacientes com a forma recorrente-remitente (EMRR), pacientes com a forma secundariamente progressiva (EMSP), com surtos ou primariamente progressiva (EMPP), pode ser feito o tratamento através de transplante autólogo de células-tronco hematopoiéticas (TACTH), usando as próprias células do paciente, melhorando as condições inflamatórias para malignidade hematológica

\section{REFERÊNCIAS}

DÍAZ, C., Zarco, L.A., Rivera, D.M. Highly active multiple sclerosis: An update. Mult Scler Relat Disord. n. 30, págs. 215-224, 2019 doi: 10.1016/j.msard.2019.01.039. DOBSON, R., Giovannoni, G. Multiple sclerosis - a review. Eur J Neurol. 2019 n. 26 v.1 págs. 27-40. doi: 10.1111/ene.13819.

DOSHI, A., Chataway, J. Multiple sclerosis, a treatable disease. Clin Med (Lond). n. 16, e6, págs. 53-59, 2016 doi: 10.7861/clinmedicine.

KARIMI, A., Bahrampour, K., Momeni, M.M.A., Asadikaram, G., Ebrahimi, G., Torkzadeh-Mahani, M., Esmaeili, T. M., Nematollahi, M.H. Evaluation of lithium serum level in multiple sclerosis patients: A neuroprotective element. Mult Scler Relat Disord. n. 17, págs. 244-248, 2017 doi: 10.1016/j.msard.2017.08.019.

KATZ, S.I. Classification, diagnosis, and differential diagnosis of multiple sclerosis. Curr Opin Neurol. n. 28, v. 3, págs.193-205, 2015 doi: 10.1097/WCO.0000000000000206.

LEMUS, H.N., Warrington, A.E., Rodriguez, M. Multiple Sclerosis: Mechanisms of 
Disease and Strategies for Myelin and Axonal Repair. Neurol Clin. n. 36, v. 1 págs 1-11, 2018 doi: 10.1016/j.ncl.2017.08.002.

LIU, B., Xin, W., Tan, J.R., Zhu, R.P., Li, T., Wang, D., Kan, S.S., Xiong, D.K. Myelin sheath structure and regeneration in peripheral nerve injury repair. Proc Natl Acad Sci U S A. n. 29, v. 116 e44 págs. 22347-22352, 2019 doi: 10.1073/pnas.1910292116.

LORETTU, L., Carpita, B., Nivoli, A., Milia, P., De Iorio, G., Cremone, I.M., Dell'Osso, L. Lithium Use During Pregnancy in a Patient With Bipolar Disorder and Multiple Sclerosis. Clin Neuropharmacol. n. 43, v. 5, págs. 158-161, 2020 doi: 10.1097/WNF.0000000000000407.

MORSHEDI, M., Hashemi, R., Moazzen, S., Sahebkar, A., Hosseinifard, E.S. Immunomodulatory and anti-inflammatory effects of probiotics in multiple sclerosis: a systematic review. J Neuroinflammation. n. 21, v. 16 e1 págs.231-249, 2019 doi: 10.1186/s12974-019-1611-4.

RINKER, J.R. Meador, W.R., King, P. Randomized feasibility trial to assess tolerance and clinical effects of lithium in progressive multiple sclerosis. Heliyon. n. 28, v. 6 e04528, 2020 doi: 10.1016/j.heliyon.2020

SANTOS, V.S. Esclerose múltipla. Mundo educação, 2019

STADELMANN, C., Timmler, S., Barrantes-Freer, A., Simons, M. Myelin in the Central Nervous System: Structure, Function, and Pathology. Physiol Rev. n. 1 v. 3 págs. 1381-1431, 2019 doi: 10.1152/physrev.00031.2018 